\title{
Factores asociados con la incidencia de dengue en Costa Rica
}

\author{
Nelson Mena, ${ }^{1}$ Adriana Troyo, ${ }^{1}$ Roger Bonilla-Carrión ${ }^{2}$ \\ y Ólger Calderón-Arguedas ${ }^{1}$
}

Forma de citar Mena N, Troyo A, Bonilla-Carrión R, Calderón-Arguedas Ó. Factores asociados con la incidencia de dengue en Costa Rica. Rev Panam Salud Publica. 2011;29(4):234-42.

RESUMEN Objetivo. Determinar el grado de influencia de diferentes variables socioeconómicas, demográficas, geográficas y climáticas con respecto a la incidencia de dengue y dengue hemorrágico (D/DH) en Costa Rica durante el período 1999-2007.

Métodos. Se realizó un estudio epidemiológico de tipo correlacional, analizando la incidencia acumulada de D/DH desde 1999 a 2007 y su asociación con diferentes variables en los 81 cantones del país. La información se obtuvo de fuentes secundarias y las variables independientes usadas para el análisis se seleccionaron considerando su representatividad en cuanto a aspectos sociodemográficos, ambientales y de cobertura sanitaria que afectan a la epidemiología de D/DH. Estas variables se organizaron en cuatro grupos de indicadores: demográficos, socioeconómicos, de vivienda y climáticos y geográficos. Los datos se analizaron por medio de regresiones de Poisson simples y múltiples.

Resultados. Los cantones de Costa Rica con mayor incidencia de D/DH se localizaron principalmente cerca de las costas, coincidiendo con algunas de las variables estudiadas. La temperatura, la altitud y el indice de pobreza humana (IPH) fueron las variables más relevantes para explicar la incidencia de $\mathrm{D} / \mathrm{DH}$, en tanto que la temperatura fue la más significativa en los análisis múltiples.

Conclusiones. Los análisis permitieron relacionar una mayor incidencia de $D / D H$ con cantones de menor altitud, mayor temperatura y un IPH elevado. Esta información es relevante como un primer paso para estratificar prioridades y optimizar acciones de prevención y control de esta enfermedad.

Palabras clave Dengue; fiebre hemorrágica dengue; infecciones por arbovirus; incidencia; factores socioeconómicos; Costa Rica.

El dengue es la arbovirosis con mayor morbilidad y mortalidad en todo el planeta (1). Aproximadamente un tercio de la población mundial se encuentra en riesgo de adquirirla, aunque su mayor

\footnotetext{
1 Centro de Investigación en Enfermedades Tropicales, Departamento de Parasitología, Facultad de Microbiología, Universidad de Costa Rica. San José, Costa Rica. La correspondencia se debe dirigir a Adriana Troyo, adriana.troyo@ucr.ac.cr

2 Centro Centroamericano de Población y Escuela de Estadística, Universidad de Costa Rica. San José, Costa Rica.
}

incidencia se da principalmente en zonas tropicales y subtropicales (2). Su virus - de la familia Flaviviridae- presenta cuatro serotipos (DEN-1, DEN-2, DEN-3 y DEN-4) que son los responsables de los cuadros clínicos dengue clásico, dengue hemorrágico y síndrome de choque por dengue (2). En América Latina el dengue y el dengue hemorrágico (D/DH) están registrando avances que plantean crecientes desafíos a los sistemas de salud (3). Según un estudio de 2008 sobre el desarrollo humano sostenible en Centro- américa, el dengue constituye uno de los problemas de salud pública emergentes de mayor relevancia en esta región, para el cual todavía no se ha logrado una reducción sostenida en el número de casos pese a la implementación de medidas para su control (4).

En Costa Rica tal situación es relativamente reciente, pues el vector había sido erradicado en 1960 gracias al Programa de Control de Insectos del Ministerio de Salud en convenio con la Organización Panamericana de la Salud (OPS). La rein- 
troducción del mosquito se dio a principios de la década de 1990, pero los primeros casos de dengue se registraron en 1993 en las ciudades de Liberia y Puntarenas, extendiéndose posteriormente a otras zonas del país $(5,6)$. Según datos del Ministerio de Salud, desde ese año se han notificado cerca de 226000 casos de $\mathrm{D} / \mathrm{DH}$, que incluyen aproximadamente 120000 casos acumulados entre $2005 \mathrm{y}$ 2010 (7). Los 37798 casos registrados solo en 2005 ubicaron a Costa Rica como el país de mayor incidencia en América Latina (4). En 2007 y 2008 ocupó el segundo lugar de Centroamérica, solamente superado por Honduras (8).

$\mathrm{El}$ incremento en los casos de D/DH en Costa Rica podría obedecer a una serie de factores entre los que se incluyen un aumento en la densidad poblacional, la globalización del transporte -que permite la movilización de personas y mosquitos-y una urbanización descontrolada (9). En relación con este último factor, su impacto podría deberse a deficiencias en el acabado de las viviendas y en las condiciones de vida que propician el hacinamiento, así como insuficiencia de servicios básicos como agua potable, salud y saneamiento, todos aspectos que modulan positivamente la ocurrencia de la enfermedad, ya que propician el contacto humano-vector $(9,10)$.

En América Latina el principal vector de dengue es Aedes (Stegomyia) aegypti (Linnaeus, 1762), cuyo comportamiento y biología condicionan una alta eficiencia en la transmisión del virus y una amplia distribución en zonas urbanas (11). Al no existir una vacuna disponible, la mejor alternativa para prevenir la enfermedad consiste precisamente en el control vectorial. Dado que en las últimas décadas las estrategias de control y prevención no han mostrado efectos satisfactorios o no se han empleado de manera adecuada, es perentorio evaluarlas y rediseñarlas para reducir la epidemia y eventualmente eliminar esta preocupante enfermedad (12).

La incidencia de D/DH está regulada por interacciones entre el vector, el virus y el ser humano en el ecosistema (13), razón por la cual los diversos factores que afectan a estos tres elementos repercuten en la dinámica de transmisión. En Costa Rica la distribución de la incidencia de D/DH no ocurre al azar, sino que más bien se encuentra localizada principalmente en determinadas regiones geográficas (14). El objetivo del presente es- tudio fue determinar el grado de influencia de diferentes variables socioeconómicas, demográficas, geográficas y climáticas con respecto a esta incidencia en los cantones del país durante el período 1999-2007. Sus resultados deberían contribuir al desarrollo de una nueva estrategia que incluya acciones efectivas contra los factores que favorecen la ocurrencia de dengue. Dadas las similitudes socioeconómicas, culturales, geográficas, climáticas y de vegetación que caracterizan a ciertas zonas de Costa Rica y de otros países latinoamericanos, se espera que los hallazgos de esta investigación ayuden a establecer comparaciones para un mayor entendimiento de esta enfermedad en la Región.

\section{MATERIALES Y MÉTODOS}

\section{Área de estudio}

Costa Rica tiene una extensión territorial de $51100 \mathrm{~km}^{2}$ —dividida políticamente en 81 cantones-y una población aproximada de 4622000 habitantes (15). La mayor parte del país presenta dos estaciones claramente definidas —una estación seca (diciembre-abril) y una estación lluviosa (mayo-noviembre) - y temperaturas que oscilan entre $7,4^{\circ} \mathrm{C}$ y $29^{\circ} \mathrm{C}$, dependiendo las zonas (16). También la frecuencia de las precipitaciones depende de las zonas, con drásticas diferencias que van desde mínimos anuales promedio de $910 \mathrm{~mm}^{3}$ a máximos de $7686 \mathrm{~mm}^{3}$ (16). En tal sentido, dada su heterogeneidad territorial en relieve, vegetación, temperatura y precipitaciones, el país cuenta con varios microclimas separados por pequeñas distancias.

\section{Variables}

La resolución para este estudio fue a nivel de cantones, considerando la disponibilidad de datos a esa escala para las variables. Como variable dependiente se utilizó la incidencia de D/DH para cada uno de los 81 cantones del país. De acuerdo al sistema de salud costarricense, los casos de D/DH confirmados por pruebas serológicas adecuadas son de notificación obligatoria. Sin embargo, ante un brote epidémico, los casos sospechosos clínicamente son considerados positivos e informados al Ministerio de Salud (17). El total acumulado de casos de $\mathrm{D} / \mathrm{DH}$ por cantón notificados durante el período 1999-2007 fue calculado a partir de los casos anuales registrados para cada cantón en la oficina de estadística del Ministerio. No se discriminó entre casos de dengue y dengue hemorrágico debido a que este último representó menos de 1\% del total (137 719) registrado en ese período (7). La población total de cada cantón en la mitad del período de estudio (año 2003) se obtuvo de las estimaciones del Centro Centroamericano de Población (CCP) y se consideró como una media temporal que serviría como variable de desplazamiento en las regresiones de Poisson (15).

Las variables independientes usadas para el análisis se seleccionaron considerando su representatividad en cuanto a aspectos sociodemográficos, ambientales y de cobertura sanitaria que afectan a la epidemiología de D/DH, además de su confiabilidad, compleción y disponibilidad. Estas variables se organizaron como indicadores en cuatro categorías: demográficos, socioeconómicos, de vivienda y climáticos y geográficos.

Indicadores demográficos. Incluyeron: 1) densidad de población, 2) porcentaje de población urbana, 3) relación de dependencia demográfica, ${ }^{3}$ 4) tasa de fecundidad general y 5) razón hombre/ mujer. Los datos fueron obtenidos del Instituto Nacional de Estadística y Censos (INEC) y corresponden a la información que generó el último censo realizado en el país en 2000 (18).

Indicadores socioeconómicos. Incluyeron: 1) índice de desarrollo humano $(\mathrm{IDH}), 2)$ índice de pobreza humana (IPH), 3) tasa de analfabetismo, 4) tasa de población con estudios secundarios o más, 5) tasa de desempleo abierto y 6) tasa de población no asegurada. Los datos de IDH e IPH se tomaron del "Atlas del desarrollo humano cantonal de Costa Rica" (19), calculando para cada cantón un promedio del período 2000-2005. Los indicadores restantes se obtuvieron de las bases de datos del INEC y el CCP y corresponden a la información del censo realizado en 2000 (18).

Indicadores de vivienda. Incluyeron las tasas de: 1) viviendas hacinadas, 2) viviendas en buen estado, 3) viviendas en mal estado, 4) viviendas con acueductos

\footnotetext{
3 Número de personas económicamente activas por cada 100 que no deberían serlo (menores de 15 años y mayores de 65 años de edad).
} 
y 5) viviendas con sanitario (alcantarilla y tanque). Estos datos se tomaron del INEC y corresponden a la información del censo realizado en 2000 (18).

Indicadores climáticos y geográficos. Incluyeron: 1) temperatura, 2) altitud, 3) precipitaciones e 4) índice de vegetación mejorado (EVI, del inglés "Enhanced Vegetation Index"). La altitud que se utilizó para el análisis correspondió a la del principal centro urbano de cada cantón (16). Dado que varios estudios han asociado los casos de D/DH con la densidad de población, en el presente trabajo se supuso que la altitud del principal centro urbano representaría adecuadamente las condiciones en el cantón de donde provienen la mayor parte de los casos notificados (20).

Los datos sobre temperatura y precipitaciones fueron recogidos de registros de diferentes estaciones del Instituto Meteorológico Nacional (IMN) y de registros históricos (16). Debido a la posibilidad de que en algunos cantones haya más de una estación o no haya datos del IMN, se decidió utilizar la información disponible de las estaciones, o en su defecto del registro histórico, considerando condiciones tales como presencia de información coincidente con el período de estudio, medición de todas las variables de interés por la misma estación y similitud de la altitud de la estación con la de los centros urbanos del cantón. Para la temperatura se empleó el promedio anual de las estaciones pertenecientes a cada cantón, en tanto que para las precipitaciones se utilizó el promedio del acumulado anual de lluvia en milímetros cúbicos brindado por las estaciones.

Los datos del EVI para los cantones fueron extraídos de la imagen satelital del sensor MODIS correspondiente al período del 10 al 25 de junio de 2003 (número 161). Para cada cantón se utilizó el promedio del EVI, que se notifica con una resolución espacial de 500 m. El EVI, como otros índices de vegetación, se relaciona con la actividad fotosintética y las variaciones estructurales del dosel. Se eligió por ser un índice más sensible que optimiza la señal reduciendo las influencias atmosféricas y del suelo y presenta una alta respuesta a variaciones fenológicas (21).

\section{Análisis de los datos}

Tras una observación preliminar del comportamiento de los datos, se crearon mapas ilustrativos para representar la información de las variables independientes y la incidencia de D/DH por cantón - calculada a partir del total acumulado de casos de D/DH durante 1999-2007 y la población estimada de cada cantón a la mitad de ese período. Los mapas se crearon utilizando el software Idrisi Taiga (Clark Labs) y con cuatro categorías para los valores, optimizando así su visualización. Las categorías fueron seleccionadas automáticamente con la técnica de intervalos iguales, excepto para las variables razón hombre/mujer, tasa de desempleo abierto, EVI e incidencia de $\mathrm{D} / \mathrm{DH}$, donde se aplicó el método de cuantiles (22).

Los análisis preliminares también incluyeron un estudio de correlaciones entre las variables independientes. Con los datos de cada variable se realizaron pruebas de normalidad (Shapiro-Wilk) empleando un nivel de significación de 0,05 para determinar la prueba de correlación adecuada (Pearson o Spearman). No se transformó ninguna de las variables independientes previo a su inclusión en los análisis.

Para el análisis estadístico posterior se supuso que los casos de $\mathrm{D} / \mathrm{DH}$ tienen una distribución de Poisson y las variables se sometieron a pruebas de bondad de ajuste al modelo de Poisson por el método de máxima verosimilitud con el programa Stata 9.0 (StataCorp) $(14,23)$. Cada variable independiente se analizó contra la incidencia en los cantones por medio de una regresión lineal de Poisson, con el fin de evaluar individualmente la asociación de cada una con la incidencia de $\mathrm{D} / \mathrm{DH}$. La regresión de Poisson es una técnica disponible para modelar variables dependientes de tipo conteo que describen datos discretos o tasas. Se supone, por ejemplo, que la variable dependiente "casos de una enfermedad que ocurren en un tiempo o en una población determinada" tiene una distribución de Poisson y que el logaritmo de su valor previsto puede ser modelado por una combinación de parámetros desconocidos. En estos modelos, los coeficientes son estimados por máxima verosimilitud y la "desviación" mide el ajuste del modelo de Poisson (23). Para las regresiones se empleó la relación entre el total de casos de D/DH normalizada por la población del cantón correspondiente a la mitad del período de estudio - esta última incluida como variable de desplazamiento. Esta rela- ción representó la tasa de incidencia de $\mathrm{D} / \mathrm{DH}$ del cantón. El nivel de significancia general para el conjunto de 20 pruebas en esta etapa se estableció en 0,1 y se ajustó por el método de Bonferroni para análisis múltiples (24). Por lo tanto, la asociación fue considerada significativa para variables cuyo coeficiente presentara una $P<0,005$.

Adicionalmente, se realizaron regresiones múltiples de Poisson con el fin de determinar la relevancia y el grado de influencia de las variables por categoría sobre la incidencia de D/DH en los cantones. Las variables independientes más relevantes en cada una de las categorías fueron seleccionadas analizando todos los indicadores de cada categoría para obtener modelos por medio de tres métodos diferentes: 1) eliminación (backward), 2) hacia adelante (forward) y 3) adición por pasos inteligentes (stepwise) (23). Los modelos con una menor desviación fueron considerados los mejores y las variables que permanecieron en esos modelos fueron reconocidas como las más importantes. En cada uno de estos análisis múltiples se estableció un nivel de significancia de 0,05.

Las variables independientes más relevantes de cada categoría, que fueron seleccionadas a partir del modelo con la menor desviación, se introdujeron en un nuevo análisis múltiple para obtener un modelo final. Los métodos para identificar las variables que permanecieron en este modelo final fueron los mismos que se emplearon en los análisis para cada categoría de indicador. En todos los análisis estadísticos donde el programa no fue especificado se utilizó el Statistix 8 Student Edition (Analytical Software).

\section{RESULTADOS}

Los cantones con mayor incidencia de $\mathrm{D} / \mathrm{DH}$ fueron los localizados principalmente cerca de las costas, tanto del Caribe como del Pacífico (figura 1). Varios de estos cantones también coincidieron en tener baja altitud y valores altos de razón hombre/mujer, IPH, analfabetismo, desempleo, temperatura y EVI (figura 2).

Los análisis exploratorios de correlaciones entre variables independientes mostraron que en su mayoría tenían algún grado de correlación (datos no presentados). Al realizar las regresiones individuales para cada variable independiente, se observó que ninguno de los in- 
FIGURA 1. Incidencia de dengue/dengue hemorrágico en los cantones de Costa Rica, 1999-2007

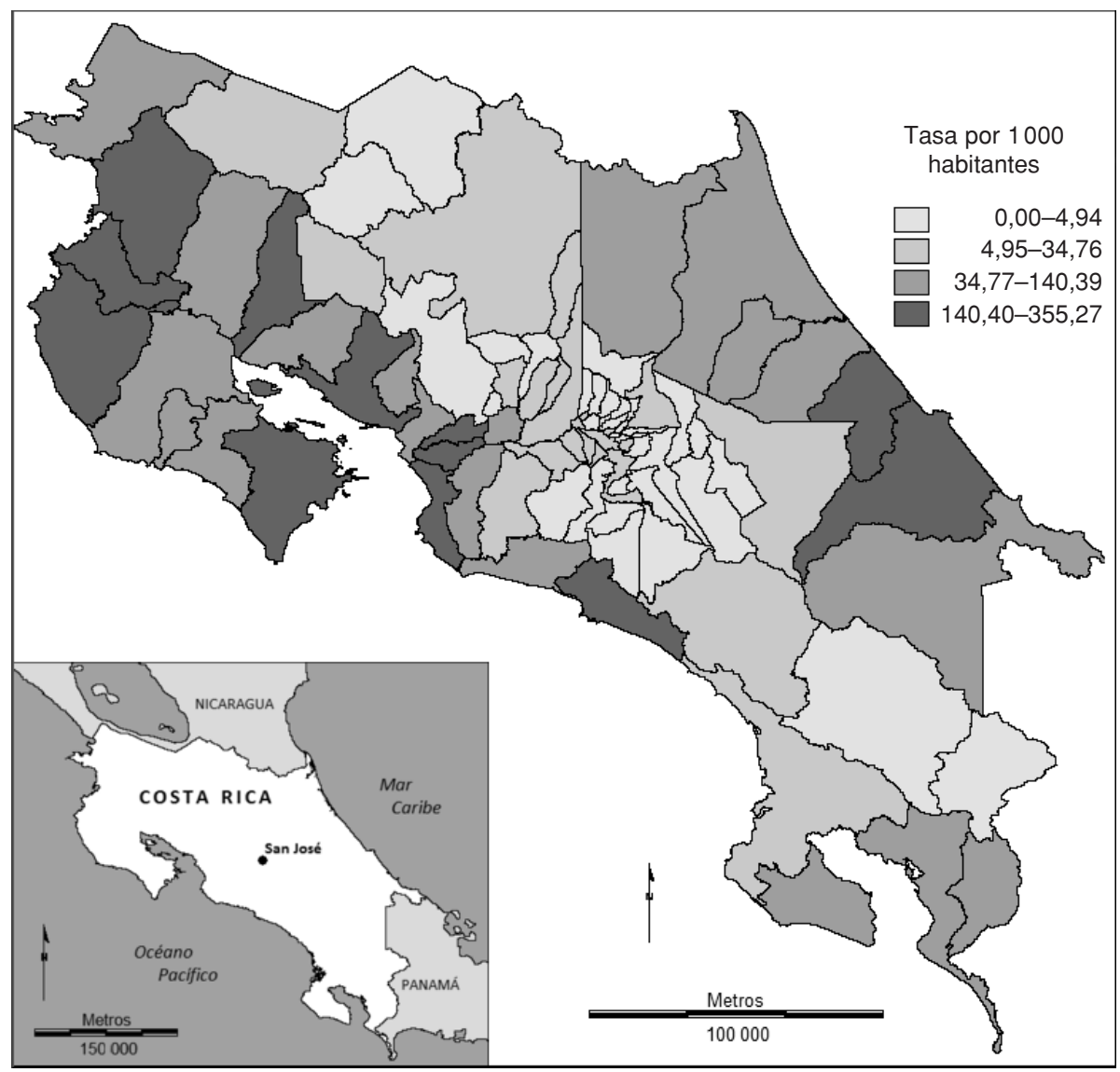

Fuente: elaboración de los autores. dicadores de vivienda se asoció significativamente con la incidencia de $\mathrm{D} / \mathrm{DH}$ en los cantones. Mientras tanto, solo la razón hombre/mujer de los indicadores demográficos se asoció significativamente con la incidencia de D/DH. En cuanto a los indicadores socioeconómicos, tanto el IPH como las tasas de analfabetismo y de desempleo abierto resultaron significativos. Con excepción de las precipitaciones, los indicadores climáticos y geográficos evaluados también fueron significativos. Asimismo, los valores más bajos de $P$ para los coeficientes y las desviaciones más bajas de los modelos se encontraron en la categoría de indicadores climáticos y geográficos, seguidos por los indicadores socioeconómicos (cuadro 1).

Las variables relacionadas con la vivienda se excluyeron de los análisis múltiples luego de observar que ninguna fue significativa en los análisis individuales. Una sola variable independiente para cada categoría se identificó en las regresiones múltiples por categoría. La $P$ del modelo fue menor a 0,0001 en todos los casos. El indicador demográfico más relevante fue la razón hombre/mujer (desviación $=29,93$; coeficiente $P=0,004)$ y la misma variable fue identificada por los tres métodos diferentes utilizados. Tanto para los indicadores socioeconómicos como los climáticos y geográficos se

FIGURA 2. Variables asociadas significativamente con la incidencia de dengue/dengue hemorrágico en los cantones de Costa Rica, 1999-2007
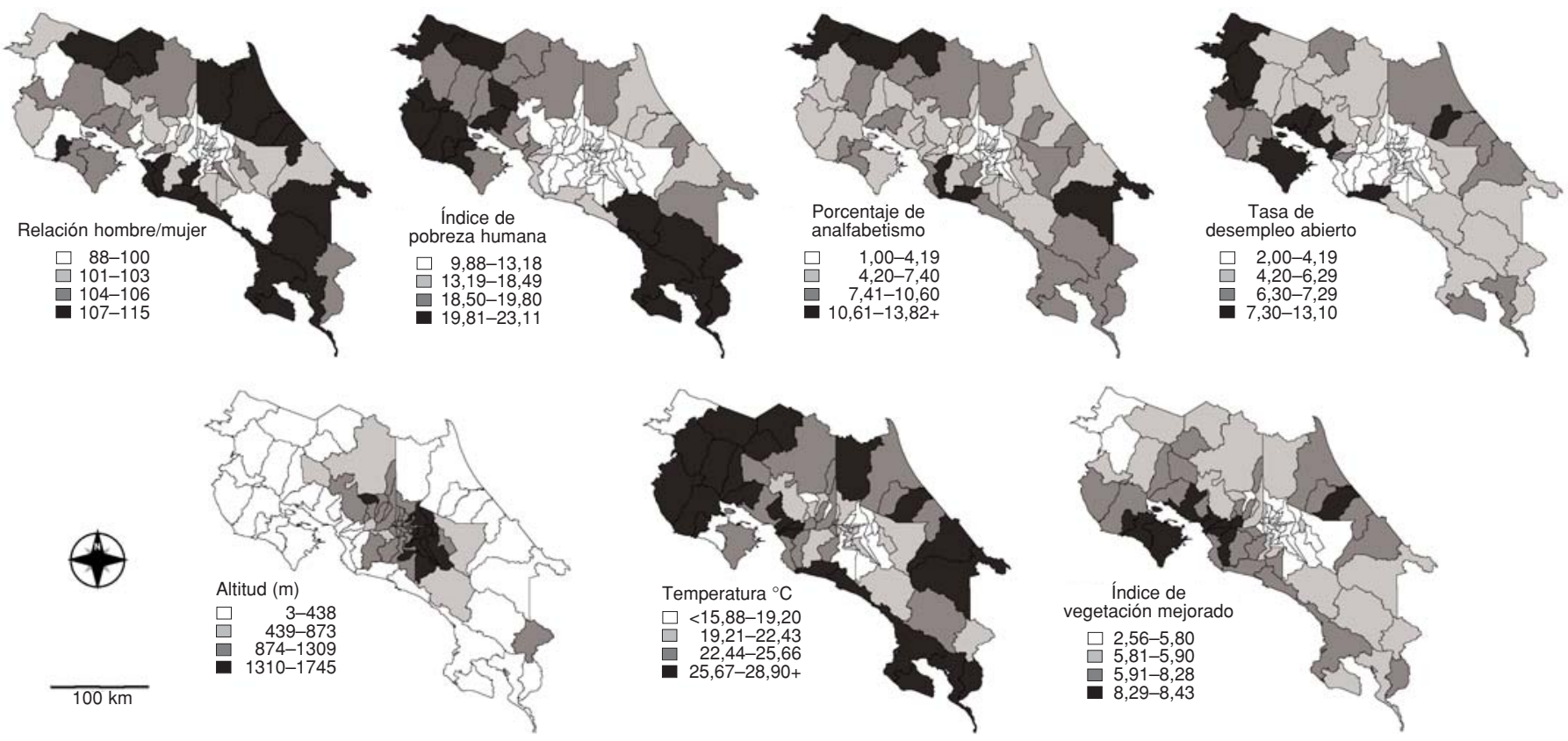

Fuente: elaboración de los autores. 
CUADRO 1. Variables asociadas con la incidencia de dengue/dengue hemorrágico en los cantones de Costa Rica, 1999-2007

\begin{tabular}{|c|c|c|c|c|c|c|}
\hline Categoría & Variable & $\begin{array}{l}\text { Razón de } \\
\text { incidencia }\end{array}$ & Coeficiente & $\begin{array}{l}\text { Error } \\
\text { estándar }\end{array}$ & $P a$ & $\begin{array}{l}\text { Desviación } \\
\text { del modelo }\end{array}$ \\
\hline \multirow{5}{*}{$\begin{array}{l}\text { Indicadores } \\
\text { demográficos }\end{array}$} & Densidad de población $/ \mathrm{km}^{2}$ & 0,999 & $-9,54 \times 10^{-5}$ & $4,90 \times 10^{-5}$ & 0,051 & 33,65 \\
\hline & Porcentaje de población urbana & 0,994 & $-0,006$ & 0,003 & 0,017 & 32,24 \\
\hline & Relación de dependencia demográfica & 1,012 & 0,012 & 0,008 & 0,120 & 35,72 \\
\hline & Tasa de fecundación general & 1,496 & 0,403 & 0,210 & 0,055 & 34,51 \\
\hline & Razón hombre/mujer & 1,035 & 0,034 & 0,012 & $0,004^{b}$ & 29,93 \\
\hline \multirow{6}{*}{$\begin{array}{l}\text { Indicadores } \\
\text { socioeconómicos }\end{array}$} & Índice de desarrollo humano & 0,203 & $-1,596$ & 1,017 & 0,116 & 35,63 \\
\hline & Índice de pobreza humana & 1,048 & 0,046 & 0,015 & $0,002^{b}$ & 28,75 \\
\hline & Porcentaje de analfabetismo & 1,060 & 0,058 & 0,021 & $0,005^{b}$ & 30,57 \\
\hline & Porcentaje de población no asegurada & 1,014 & 0,013 & 0,012 & 0,239 & 36,80 \\
\hline & $\begin{array}{l}\text { Porcentaje de población con estudios } \\
\text { secundarios o superiores }\end{array}$ & 0,990 & $-0,009$ & 0,005 & 0,049 & 34,16 \\
\hline & Tasa de desempleo abierto & 1,102 & 0,097 & 0,032 & $0,002^{b}$ & 29,59 \\
\hline \multirow{5}{*}{$\begin{array}{l}\text { Indicadores } \\
\text { de vivienda }\end{array}$} & Porcentaje de viviendas con acueducto & 0,989 & $-0,010$ & 0,005 & 0,028 & 33,52 \\
\hline & Porcentaje de viviendas en buen estado & 0,987 & $-0,013$ & 0,006 & 0,023 & 33,07 \\
\hline & Porcentaje de viviendas con hacinamiento & 0,989 & $-0,010$ & 0,005 & 0,028 & 33,52 \\
\hline & Porcentaje de viviendas en mal estado & 1,031 & 0,030 & 0,014 & 0,027 & 33,41 \\
\hline & $\begin{array}{l}\text { Porcentaje de viviendas con sanitario } \\
\quad \text { (alcantarilla o tanque) }\end{array}$ & 0,988 & $-0,012$ & 0,005 & 0,027 & 33,53 \\
\hline \multirow{4}{*}{$\begin{array}{l}\text { Indicadores } \\
\text { climáticos y } \\
\text { geográficos }\end{array}$} & Altitud & 0,999 & $-5,81 \times 10^{-4}$ & $1,31 \times 10^{-4}$ & $<0,001^{b}$ & 18,38 \\
\hline & Precipitaciones & 1,000 & $1,77 \times 10^{-5}$ & $9,14 \times 10^{-5}$ & 0,846 & 36,97 \\
\hline & Temperatura & 1,095 & 0,090 & 0,022 & $<0,001^{b}$ & 16,28 \\
\hline & Índice de vegetación mejorado & 1,337 & 0,290 & 0,103 & $0,005^{b}$ & 28,80 \\
\hline
\end{tabular}

Fuente: elaboración de los autores.

a $P$ del coeficiente.

b $P$ del coeficiente. Estos valores fueron considerados significativos con un nivel de confianza de 90\%, ajustado para los 20 análisis múltiples.

CUADRO 2. Variables identificadas como principales determinantes de la incidencia de dengue/dengue hemorrágico en los cantones de Costa Rica, por categoría, 1999-2007

\begin{tabular}{|c|c|c|c|c|c|c|c|c|}
\hline Categoría & Métodoa & Variable & Coeficiente & Constante & $\begin{array}{l}\text { Error } \\
\text { estándar }\end{array}$ & $P^{\mathrm{b}}$ & $\begin{array}{l}\text { Desviación } \\
\text { del modelo }\end{array}$ & $\begin{array}{l}\text { Grados de } \\
\text { libertad }\end{array}$ \\
\hline Indicadores demográficos & 1,2 y 3 & $\begin{array}{l}\text { Relación } \\
\text { hombre/mujer }\end{array}$ & 0,034 & $-7,160$ & 0,012 & $0,004^{c}$ & 29,93 & 78 \\
\hline \multirow[t]{2}{*}{ Indicadores socioeconómicos } & 1 & $\begin{array}{l}\text { Tasa de desempleo } \\
\text { abierto }\end{array}$ & 0,097 & $-4,121$ & 0,032 & $0,002^{c}$ & 29,59 & 78 \\
\hline & 2 y 3 & $\begin{array}{l}\text { Índice de pobreza } \\
\text { humana }\end{array}$ & 0,046 & $-4,302$ & 0,015 & $0,002^{c}$ & 28,75 & 78 \\
\hline $\begin{array}{l}\text { Indicadores climáticos y } \\
\text { geográficos }\end{array}$ & $\begin{array}{c}1 \\
2 \text { y } 3\end{array}$ & $\begin{array}{l}\text { Altitud } \\
\text { Temperatura }\end{array}$ & $\begin{array}{c}-5,8 \times 10^{-4} \\
0,090\end{array}$ & $\begin{array}{l}-3,232 \\
-5,725\end{array}$ & $\begin{array}{c}1,3 \times 10-4 \\
0,022\end{array}$ & $\begin{array}{l}<0,001^{c} \\
<0,001^{c}\end{array}$ & $\begin{array}{l}18,38 \\
16,28\end{array}$ & $\begin{array}{l}78 \\
71\end{array}$ \\
\hline Modelo final & $\begin{array}{c}1 \\
2 \text { y } 3\end{array}$ & $\begin{array}{l}\text { Altitud } \\
\text { Temperatura }\end{array}$ & $\begin{array}{c}-5,8 \times 10^{-4} \\
0,090\end{array}$ & $\begin{array}{l}-3,232 \\
-5,725\end{array}$ & $\begin{array}{c}1,3 \times 10-4 \\
0,022\end{array}$ & $\begin{array}{l}<0,001^{c} \\
<0,001^{c}\end{array}$ & $\begin{array}{l}18,38 \\
16,28\end{array}$ & $\begin{array}{l}78 \\
71\end{array}$ \\
\hline
\end{tabular}

Fuente: elaboración de los autores.

a Métodos de selección. Método 1: eliminación (backward); Método 2: hacia adelante (forward); Método 3: adición por pasos inteligentes (stepwise).

b $P$ del coeficiente.

c $P$ del coeficiente. Estos valores fueron considerados significativos con un nivel de confianza de $95 \%$.

identificaron variables diferentes según el método empleado (cuadro 2).

La tasa de desempleo abierto y el IPH figuraron como las variables socioeconómicas más relevantes, aun cuando el $\mathrm{IPH}$, obtenido mediante los métodos hacia adelante y adición por pasos inteligentes, permitió un mejor ajuste al modelo Poisson para explicar la incidencia de D/DH en los cantones del país (desviación $=28,75$; coeficiente $P=0,002$ ) . Asimismo, la altitud y la temperatura fueron los indicadores climáticos y geo- gráficos más relevantes, pero la temperatura presentó la menor desviación (desviación $=16,28$; coeficiente $P<$ 0,001).

Las variables razón hombre/mujer, tasa de desempleo, IPH, altitud y temperatura fueron incluidas en el análisis para determinar las variables independientes relevantes en el modelo final, según los resultados de los análisis por categoría. Una vez más, los diferentes métodos de selección de variables resultaron en modelos con una única variable: la altitud (desviación $=18,38$; coeficiente $P<0,001)$ o la temperatura (desviación = 16,28; coeficiente $P<0,001$ ) (cuadro 2). La $P$ de estos modelos fue menor a 0,0001 . La temperatura fue seleccionada como la variable incluida en el modelo con menor desviación mediante dos de los métodos (hacia adelante y adición por pasos inteligentes). La ecuación de este modelo final fue:

Incidencia $\mathrm{D} / \mathrm{DH}=$ $\mathrm{e}^{-5,725+0,090 \text { (Temperatura) }}$. 


\section{DISCUSIÓN}

En el presente estudio se encontró que las variables que mostraron una mayor relación con la incidencia de $\mathrm{D} / \mathrm{DH}$ por cantón en Costa Rica se ubicaron principalmente en las categorías de indicadores climáticos y geográficos, y socioeconómicos. Las variables climáticas y geográficas ejercen su efecto en aspectos como la distribución de los vectores, su ciclo de vida, su supervivencia diaria y la regulación del ciclo extrínseco viral (13). Además, el comportamiento humano, su estilo de vida y la infraestructura en que vive pueden condicionar la transmisión del dengue, como ya ha sido demostrado en diversos estudios $(1,12,25)$.

De los indicadores socioeconómicos, el IPH y la tasa de desempleo, presentaron una relación positiva con la incidencia de $\mathrm{D} / \mathrm{DH}$ en los diferentes cantones. En cuanto al desempleo, aun cuando estuvo incluido en el cálculo del IPH, se asoció claramente con la incidencia de la enfermedad al margen de los demás elementos de este índice. Sin embargo, considerando que la tasa de desempleo mostró un menor ajuste al modelo de Poisson que el IPH, este índice sería un mejor indicador para reflejar las condiciones socioeconómicas que favorecen la transmisión y la incidencia de D/DH.

Estos indicadores socioeconómicos fueron dos de los más relevantes de todo el análisis, superados solo por la temperatura y la altitud. Diversos estudios han asociado las bajas condiciones socioeconómicas con la incidencia de dengue, principalmente debido a que el riesgo de transmisión se incrementa por razones tales como servicios inadecuados de suministro de agua, mal manejo de los desechos sólidos, condiciones de urbanización deficientes y bajo nivel de educación (3).

Aunque el IDH no se relacionó con la incidencia de $\mathrm{D} / \mathrm{DH}$ en este estudio, otros autores lo han considerado apropiado para mostrar la "penetración" que los recursos financieros pueden tener en beneficio de la salud y la educación de la población (26). Algunas de las otras variables socioeconómicas analizadas forman parte de la fórmula de cálculo del IDH o explican fenómenos similares. La incidencia de D/DH se asoció con el analfabetismo presente en los cantones, aunque - como se dijo- su asociación con el IDH no fue significativa. Por lo tanto, el analfabetismo parece ser más re- levante que otros elementos incluidos en el cálculo del IDH, como el nivel de escolaridad.

En el presente estudio la tasa de analfabetismo permitió explicitar el efecto de la baja escolaridad en la incidencia de D/DH. Otros trabajos realizados en Honduras y México demostraron una relación positiva entre criaderos positivos por A. aegypti y poca escolaridad de las madres de familia $(25,27)$. El bajo nivel educativo de una región podría dificultar el entendimiento real del riesgo que representa el dengue para los habitantes, reduciendo al mismo tiempo los conocimientos básicos relacionados con el vector que son necesarios para su control (27). Por otro lado, la continuidad de la educación — reflejada en la tasa de población con estudios secundarios o superiores- no se asoció con la incidencia de $\mathrm{D} / \mathrm{DH}$, lo cual sugiere que esta enfermedad solo se relaciona con un nivel muy bajo educación.

La tasa de población sin seguro de salud fue otro de los indicadores socioeconómicos que no logró explicar la incidencia de $\mathrm{D} / \mathrm{DH}$ en los cantones de Costa Rica. Con esta variable se pretendía evaluar la cobertura de los servicios de salud, ya que actualmente se considera que su detrimento en términos de calidad y acceso ha perjudicado los programas de erradicación y control de A. aegypti en América Latina y el Caribe $(2,20)$. Este panorama ha favorecido la endemicidad y la incidencia de la enfermedad en algunas zonas de la Región (28). En este trabajo, sin embargo, la ausencia de relación entre D/DH y la población asegurada podría explicarse porque en Costa Rica el sistema de salud aún presenta un carácter social y tiende a la universalidad en su cobertura. La variable población no asegurada, no obstante, si bien se correlacionó con variables que describen pobreza como el IPH, el desempleo y las viviendas en mal estado (datos no mostrados), no fue capaz de explicar por sí misma la incidencia de $\mathrm{D} / \mathrm{DH}$.

La asociación directa observada entre dicha incidencia y la razón hombre/ mujer contrasta con información de otros países como Brasil y México, pero concuerda con datos de otros lugares como India y Singapur, donde se ha evidenciado una mayor incidencia en hombres que en mujeres similar a la documentada por el Ministerio de Salud de Costa Rica $(3,7,29,30)$. Algunos autores relacionan este hallazgo con la labor típicamente asociada a cada sexo, cuyo espacio de ejecución expone a hombres y mujeres a riesgos distintos de transmisión (31).

Otras variables analizadas fueron los indicadores de vivienda, relacionados en otros estudios con factores que afectan a la transmisión del dengue, tales como la disponibilidad de criaderos y la facilidad de ingreso del vector a la vivienda (13, $32,33)$. Según el nivel de significancia estadística establecido para las regresiones individuales, en este trabajo ninguno de los indicadores de vivienda se asoció con la incidencia de D/DH. Sin embargo, las malas condiciones de las viviendas se han asociado con casos de dengue y criaderos positivos por $A$. aegypti en otros países (29). Asimismo, la relación inversa entre la incidencia de $\mathrm{D} / \mathrm{DH}$ y el porcentaje de viviendas con acueducto - aunque no significativa - podría a la vez reflejar su relación con la necesidad de almacenar agua.

Un aspecto importante para considerar es la calidad del servicio de agua, ya que un suministro discontinuo promovería el almacenamiento de agua incluso en viviendas con acueducto, aunque por otro lado existe la posibilidad de que hábitos culturales promuevan el almacenamiento de agua sin necesidad $(34,35)$. Estudios realizados en Costa Rica y otros países de América Latina han mostrado la relevancia del almacenamiento de agua, dado que su potencial como hábitat de criaderos de A. aegypti lo convierte en un factor de riesgo para brotes de dengue aun en condiciones que, de otra manera, podrían no serle favorables (20, 33-35).

En cuanto a los indicadores climáticos y geográficos, tanto la altitud como la temperatura y el EVI lograron explicar individualmente la incidencia de $\mathrm{D} / \mathrm{DH}$ en los cantones del país. La mayor incidencia se presentó en cantones de menor altitud y temperatura más elevada, siendo un hecho conocido que las zonas que notifican mayor cantidad de casos en Costa Rica son regiones calientes y cercanas a la costa $(7,36)$. Similar a lo que ocurre en Costa Rica, en una ciudad de México se encontró que la mayoría de casos de dengue se presentaban a una altitud menor a los $600 \mathrm{msnm}$ (37).

Con respecto a la temperatura, en Indonesia se la ha relacionado con la ocurrencia de casos de dengue y en Argentina con la abundancia del vector (38, 
39). Es bien conocido el impacto de la temperatura sobre el ciclo de vida del vector, así como en el proceso multiplicativo que realiza el virus dentro del vector $(9,13)$. En diversos estudios se ha informado que una alta temperatura es capaz de acortar la duración del ciclo gonadotrófico y del ciclo extrínseco viral, además de facilitar la supervivencia del mosquito e incrementar la cantidad de alimentaciones que realiza el vector (9, 13). En el caso de Costa Rica, los cantones con mayor incidencia de $\mathrm{D} / \mathrm{DH}$ suman, a su más alta temperatura, otras condiciones como pobreza y falta de planificación urbana, que favorecen todavía más la transmisión.

Las precipitaciones fueron el único indicador climático y geográfico investigado que no logró explicar la incidencia de $\mathrm{D} / \mathrm{DH}$ entre los cantones de Costa Rica. Diversos estudios han demostrado un papel dual de las precipitaciones según la situación en regiones determinadas $(13,40)$. En Costa Rica y otros países de la región, el aumento de casos se asocia principalmente con la estación lluviosa, debido generalmente a una mayor disponibilidad de criaderos que facilita la proliferación del vector $(9,34,35)$. Sin embargo, en Costa Rica hay una amplia diversidad de patrones de precipitación y el papel de esta variable podría ser superado por otros factores ambientales y socioeconómicos, como por ejemplo cantones con alta precipitación pero bajas temperaturas y, por ende, pocos casos. Además, se ha demostrado la presencia de brotes epidémicos cuando la precipitación es mínima (época seca), es decir debido a otras condiciones que favorecen la transmisión. Por ejemplo, en Bangkok y en Puerto Rico se han visto epidemias de dengue independientes del grado de precipitación, en zonas donde el almacenamiento de agua es un factor determinante que ha explicado mejor la ocurrencia de casos $(13,38)$. En Costa Rica, estudios realizados durante la estación seca han demostrado la presencia de larvas de $A$. aegypti en recipientes utilizados para almacenar agua $(35,41)$.

La asociación entre el EVI de los cantones y la incidencia de D/DH observada podría estar relacionada con la presencia de árboles y áreas verdes en los ambientes humanos donde se desarrolla el vector (42). Adicionalmente, otros indicadores de vegetación, como el índice diferencial de vegetación norma- lizado (NDVI, por sus siglas en inglés), se han asociado con la abundancia del vector en la Gran Puntarenas, Costa Rica (42). Aunque las zonas boscosas se asocian inversamente con la presencia de A. aegypti, la vegetación presente en áreas donde existe el mosquito puede favorecer la supervivencia de los adultos al brindarles sitios de reposo. Además, permite una mayor productividad en los criaderos, proporcionándoles materia orgánica, así como protección ante la irradiación solar directa y la evaporación (43).

Aunque muchas de las variables se asociaron individualmente a la incidencia de D/DH, es conocido que la incidencia de D/DH es un proceso multicausal. Este estudio se limitó al análisis de una serie de variables, pero existen otros elementos que pueden afectar a la ocurrencia de esta enfermedad y que no fueron considerados, incluyendo aspectos de la inmunidad de las poblaciones (p. ej. seropositividad, variación en la respuesta inmune y severidad), diferentes serotipos y genotipos circulantes, casos importados, medidas de control del vector e índices entomológicos (p. ej. índices de Breteau, contenedores, viviendas y pupales) para las diferentes zonas del país. Algunos datos concernientes a estos factores se encuentran de manera fragmentaria en documentos estatales o en trabajos de investigación, pero no representan a todas las zonas del país o no son confiables. En general, la introducción de estas otras variables ayudaría a comprender el comportamiento y la distribución del dengue desde una perspectiva más amplia, donde se incluyan más componentes involucrados en la transmisión y la propagación de la enfermedad.

Dado su carácter correlacional, el presente estudio representa una primera aproximación de análisis epidemiológico aplicado a Costa Rica como país. Si bien no incluyó pruebas específicas de autocorrelación espacial, en las figuras presentadas se puede observar la incidencia de dengue en los diferentes cantones. En estudios futuros, sin embargo, se podrían incluir análisis espaciales para el desarrollo de modelos determinísticos y de estadística espacial con mayor detalle. Además, hay que considerar que las fluctuaciones en la población de los cantones en el tiempo, el subregistro (p. ej. asintomáticos), el sobrerregistro o casos importados podrían afectar el cálculo de la incidencia (20).
Por otro lado, debido a que este tipo de estudio requiere agregar los datos por cantón, siempre existe la posibilidad de que se presenten falacias ecológicas, es decir sesgos que pueden darse en razón de que las asociaciones observadas entre variables a nivel agregado no necesariamente representan a las que existen a nivel individual (44). Al no poder discernirse las variaciones (p. ej. geográficas o socioeconómicas) que se presentan local o individualmente dentro de un mismo cantón, las relaciones entre variables halladas podrían ser diferentes a las resultantes de una escala más fina (p. ej. distritos, localidades, barrios o viviendas). Por lo tanto, los resultados también revelan la necesidad de investigaciones a escalas más detalladas con el fin de lograr una mejor caracterización epidemiológica de la enfermedad y un diseño adecuado de campañas sostenibles para combatirla.

De todas las variables, la temperatura, la altitud y el IPH fueron las más relevantes. Adicionalmente, una sola fue suficiente en el modelo final, posiblemente debido en gran parte a sus correlaciones. Se resalta de los resultados que la temperatura se puede considerar como la variable que mejor explica las diferencias en la incidencia de $\mathrm{D} / \mathrm{DH}$ entre los distintos cantones de Costa Rica, ya que fue capaz de ajustar el modelo por sí misma. Según la razón de incidencias para este modelo, una diferencia de $1^{\circ} \mathrm{C}$ en la temperatura podría asociarse con una variación de la incidencia de $\mathrm{D} / \mathrm{DH}$ de 9,5\% en la misma dirección.

Es interesante resaltar el panorama epidemiológico obtenido en este estudio, en el cual se observa una mayor incidencia en cantones de menor altitud y mayor temperatura e IPH, y donde se podrían señalar zonas de riesgo implícito. Entre los cantones más afectados por $\mathrm{D} / \mathrm{DH}$ se incluyen algunos cercanos a las costas, donde se ubican ciudades importantes como Liberia, Puntarenas y Limón. En estos lugares se podrían concentrar los mayores esfuerzos y canalizar los recursos para prevenir epidemias de $\mathrm{D} / \mathrm{DH}$, así como conocer el impacto en la enfermedad que podrían tener eventuales cambios en las variables estudiadas.

El análisis de las características de los cantones de Costa Rica en función de la incidencia de $\mathrm{D} / \mathrm{DH}$ podría permitir ajustar las actividades de prevención y control a las condiciones prevalecientes en cada caso. Del estudio se desprende la 
importancia de tomar en cuenta aspectos fundamentales del espacio físico, características de la población, y el entorno ambiental y geográfico de las diferentes zonas donde se van a desarrollar campañas contra el dengue. Las políticas se deben diseñar desde un enfoque integral, en el cual el plan de trabajo se adapte a las condiciones específicas de la comunidad. La relevancia de adecuar las actividades al entorno donde tienen lugar ya ha sido revelada por otros estudios que, al menos en parte, atribuyeron el fracaso en las acciones de prevención y control al uso de un mismo tipo de campaña para todas las poblaciones, sin considerar su heterogeneidad (20).

Agradecimientos. Los autores agradecen a Adrián Avendaño y Laya Hun por sus críticas, comentarios y sugerencias al presente trabajo. Dan las gracias también a Clyde McCoy, Mary Comerford, Rosario Achí y demás participantes del Taller de Publicaciones Científicas del Instituto Nacional de Investigaciones en Salud, y a Silvia Araya, por sus valiosas observaciones y sugerencias durante la preparación del manuscrito.

\section{REFERENCIAS}

1. Guzmán M, Kourí G. Dengue: an update. Lancet Infect Dis. 2002;2:33-40.

2. Gubler DJ. Dengue and dengue hemorrhagic fever. Clin Microbiol Rev. 1998;11:480-96.

3. San Martin J, Brathwaite O, Zambrano B, Solórzano J, Bouckenooghe A, Dayan G, et al. The epidemiology of Dengue in the Americas over the last three decades: A worrisome reality. Am J Trop Med Hyg. 2010;82(1):128-35.

4. Programa Estado de la Nación, Costa Rica. Estado de la Región en Desarrollo Humano Sostenible un informe desde Centroamérica y para Centroamérica 2008. San José: Programa Estado de la Nación; 2008.

5. World Health Organization. Outbreak of classic dengue, Costa Rica. Wkly Epidemiol Rec. 1994;69:85-6.

6. Guzmán MG, Huelva G, Sáenz E, Quiroz E, De los Reyes J, Balmaseda A, et al. Reintroducción del dengue 3 en las Américas: 1994-1996. Arch Venez Med Trop Parasitol Med. 1998;2(1):8-19.

7. Ministerio de Salud, Costa Rica. Vigilancia de la Salud. San José, Costa Rica. Disponible en: http://www.ministeriodesalud.go.cr/. Acceso el 9 de febrero de 2011.

8. Organización Panamericana de la Salud. Number of reported cases of dengue \& dengue haemorrhagic fever (DHF), Region of the Americas (by country and subregion). Disponible en: http://www.paho.org/ english/ad/dpc/cd/Dengue.htm. Acceso el 07 de enero de 2011.

9. Kuno G. Review of the factors modulating dengue transmission. Epidemiol Rev. 1995; 17:321-35.

10. Guzmán MG, Kourí G, García G. El dengue y el dengue hemorrágico: prioridades de investigación. Rev Panam Salud Publica. 2006;19 (3):204-15.

11. Organización Panamericana de la Salud. Dengue y dengue hemorrágico en las Américas. Washington D.C.: OPS; 1997. (Publicación Científica 548).

12. Lloyd LS, Winch P, Ortega-Canto J, Kendall C. The design of a community-based health education intervention for the control of Aedes aegypti. Am J Trop Med Hyg. 1994;50 (4):401-11.

13. Halstead S. Dengue Virus-Mosquito Interactions. Annu Rev Entomol. 2008;53:273-91.

14. Bonilla R. Distribución espacio-temporal de la fiebre dengue en Costa Rica. Población y Salud en Mesoamérica. 2006;3(2):2. Disponible en: http://ccp.ucr.ac.cr/revista/volumenes/ 3/3-2/3-2-2/3-2-2.pdf. Acceso el 07 de enero de 2011.

15. Centro Centroamericano de Población, Costa Rica. Censos. Disponible en: http://ccp.ucr. ac.cr. Acceso el 07 de enero de 2011.

16. Chinchilla E. Atlas cantonal de Costa Rica. San José: Instituto de Fomento y Asesoría Municipal; 1987.

17. Comisión Técnica Interinstitucional, Ministerio de Salud, Costa Rica. Normas técnicas para el control del Dengue y el Dengue Hemorrágico. San José: Ministerio de Salud; 2000.

18. Instituto Nacional de Estadística y Censos, Costa Rica. Censos. Disponible en: http:// www.inec.go. cr/. Acceso el 9 de febrero 2011.

19. Programa de las Naciones Unidas para el Desarrollo-UCR. Atlas del desarrollo humano cantonal de Costa Rica. San José: EDISA; 2007.

20. Barrera R, Delgado N, Jiménez M. Estratificación de una ciudad hiperendémica en dengue hemorrágico. Rev Panam Salud Publica. 2002;8(4):225-33.

21. Huete A, Didan K, Miura T, Rodríguez E, Gao $X$, Ferreira L. Overview of the radiometric and biophysical performance of the MODIS vegetation indices. Remote Sens Environ. 2002;83:195-213.

22. Eastman JR. Idrisi Taiga. Worcester: Clark University; 2009.

23. Kleinbaum D, Kupper L, Muller K, Nizam A. Applied Regression Analysis and Other Multivariable Methods. Pacific Grove: Brooks/ Cole Publishing Company; 1998.

24. Shott S. Statistics for health professionals. Philadelphia: W.B. Saunders Company; 1990.

25. Danis-Lozano R, Rodríguez M, HernándezÁvila M. Gender-related family head schooling and Aedes aegypti larval breeding risk in southern Mexico. Salud Publica Mex. 2002;44: $237-42$.

26. Rodriguez-Contreras P. Situación socio-sanitaria en Iberoamérica. Indicadores de salud y desarrollo. Ars Pharmaceutica. 2000;41(3): 325-38.

27. Ávila G, Martínez M, Sherman C, Fernandez E. Evaluación de un módulo escolar sobre dengue y Aedes aegypti dirigido a escolares en Honduras. Rev Panam Salud Publica. 2004;16(2):84-94.

28. Schmunis A, Pinto J. La reforma del sector salud, descentralización, prevención y control de enfermedades transmitidas por artrópodos. Cad Saude Pub. 2000;16(2 supl):117-23.
29. Guha-Sapir D, Schimmer B. Dengue fever: new paradigms for a changing epidemiology. Emerg Themes Epidemiol. 2005;2:1-10.

30. Goh KT. Dengue a re-emerging infectious disease in Singapore. Ann Acad Med Singapore. 1997;26:664-70.

31. Ooi EE. Changing pattern of dengue transmission in Singapore. Dengue Bull. 2001;25:40-4.

32. Getis A, Morrison A, Gray K, Scott T. Characteristics of the spatial pattern of the dengue vector, Aedes aegypti, in Iquitos, Peru. Am J Trop Med Hyg. 2003;69:494-505.

33. Morrison A, Gray K, Getis A, Astete H, Sihuincha M, Focks D, et al. Temporal and geographical patterns of Aedes aegypti (Diptera: Culicidae) production in Iquitos, Peru. J Med Entomol. 2004;41(6):1123-42.

34. Barrera R, Navarro JC, Mora Rodríguez JD, Dominguez D, González García JE. Deficiencia en servicios públicos y cría de Aedes aegypti en Venezuela. Bol Oficina Sanit Panam. 1995;118(5):410-23.

35. Troyo A, Calderon-Arguedas O, Fuller DO, Solano ME, Avendaño A, Arheart KL, et al. Seasonal profiles of Aedes aegypti (Diptera: Culicidae) larval habitats in an urban area of Costa Rica with a history of mosquito control. J Vector Ecol. 2008;33(1):76-88.

36. Marín R, Marquetti M, Álvarez Y, Gutiérrez J, González R. Especies de mosquitos (Diptera: Culicidae) y sus sitios de cría en la región Huetar Atlántica, Costa Rica. Rev Biomed. 2009;20:15-23.

37. Escobar-Mesa J, Gómez-Dantés H. Determinantes de la transmisión de dengue en Veracruz: un abordaje ecológico para su control. Salud Publica Mex. 2003;45:43-53.

38. Arcari P, Tapper N, Pfuller S. Regional variability in relationships between climate and dengue/DHF in Indonesia. Singap J Trop Geogr. 2007;28:251-72.

39. Vezzani D, Velásquez $S$, Schweigmann $\mathrm{N}$. Seasonal Pattern of Abundance of Aedes aegypti (Diptera: Culicidae) in Buenos Aires City, Argentina. Mem Inst Oswaldo Cruz. 2004;99(4):351-6.

40. Patz J, Martens W, Focks D, Jetten T. Dengue fever epidemic potential as projected by general circulation models of global climate change. Environ Health Perspect. 1998;106 (3):147-52.

41. Calderón-Arguedas O, Troyo A, Solano ME. Sitios potenciales para la multiplicación de Aedes aegypti (Diptera: Culicidae) en relación con las características de las viviendas de la 
comunidad "La Carpio", San José, Costa Rica durante la estación seca de 2003. Rev Costarric Salud Publica. 2003;12:22-30.

42. Troyo A, Fuller D, Calderon-Arguedas O, Solano M, Beier J. Urban structure and dengue incidence in Puntarenas, Costa Rica. Singap J Trop Geogr. 2009;30(2):265-82.
43. Bisset-Lazcano J, Marquetti M, Portillo R, Rodriguez M, Suarez S, Leyva M. Factores ecológicos asociados con la presencia de larvas de Aedes aegypti en zonas de alta infestación del municipio Playa, Ciudad de La Habana, Cuba. Rev Panam Salud Publica. 2006;19(6):379-84.
44. Friis RH, Sellers TA. Epidemiology for Public Health Practice. 3rd ed. Sudbury: Jones and Bartlett Publishers; 2004.

Manuscrito recibido el 23 de abril de 2010. Aceptado para publicación, tras revisión, el 23 de noviembre de 2010.

ABSTRACT Objective. Determine the extent to which socioeconomic, demographic, geographic, and climate variables affected the incidence of dengue and dengue hemorrhagic fever (D/DH) in Costa Rica during the period 1999-2007.

Factors associated with incidence of dengue in Costa Rica

Key words
Methods. A correlational epidemiologic study was conducted that analyzed the cumulative incidence of D/DH from 1999 to 2007 and its association with different variables in the country's 81 cantons. Information was obtained from secondary sources, and the independent variables used for the analysis were selected on the basis of their representativeness in terms of sociodemographic, environmental, and health coverage factors that affect the epidemiology of D/DH. These variables were divided into four groups of indicators: demographic, socioeconomic, housing, and climate and geographical. The data were analyzed by means of simple and multiple Poisson regressions.

Results. The Costa Rican cantons with a higher incidence of D/DH were located primarily near the coast, coinciding with some of the variables studied. Temperature, altitude, and the human poverty index were the most relevant variables in explaining the incidence of $\mathrm{D} / \mathrm{DH}$, while temperature was the most significant variable in the multiple analyses.

Conclusions. The analyses made it possible to correlate a higher incidence of D/DH with lower-altitude cantons, higher temperature, and a high human poverty index ranking. This information is relevant as a first step toward prioritizing and optimizing actions for the prevention and control of this disease.

Dengue; dengue hemorrhagic fever; arbovirus infections; incidence; socioeconomic factors; Costa Rica. 